\title{
Numerical Simulation of the Oscillatory Ventilation in Simplified Human Lung Models
}

\author{
Daniel Feldmann and Claus Wagner
}

\begin{abstract}
To improve the understanding of gas transport mechanisms under conditions of high-frequency oscillatory ventilation (HFOV), we performed numerical simulations of the unsteady flow in simplified airway geometries using, time varying Dirichlet boundary conditions (BC) for the pressure. The flow in a generic trachea shows excellent agreement with the analytical solution for non-dimensional frequencies up to $W o=14$. Nonetheless, the computational effort increases with Wo due to a long relaxation phase and strong velocity gradients near the wall despite of relatively low Reynolds numbers. We also use the pressure BCs on multiple open outlets at a single bifurcation and a three-generation airway model, while guaranteeing mass conservation. By employing identical and slightly different pressure values at the distal bifurcation ends, flow fields are reproduced, similar to that occurring under conditions of uneven physiological behaviour of different lung regions.
\end{abstract}

\section{Introduction}

High-frequency oscillatory ventilation (HFOV) is an artificial respiration technique employing "breathing" frequencies up to $15 \mathrm{~Hz}$ and low tidal volumes, usually below the anatomical dead space. It is stated to reduce ventilator associated lung injury from overdistension of lung epithelium due to high tidal volumes, excessive alveolar pressure values as well as cyclic alveolar re-collapse and resulting inflammatory response. Therefore, HFOV has great potential to improve clinical results from acute respiratory distress syndrome (ARDS), see e.g. Krishnan \& Brower [6]. However, advancing HFOV from being a rescue means to being an accepted alternative ventilation strategy certainly requires detailed knowledge of the governing transport mechanisms, which highly differ from those in spontaneous breathing. To gain

Daniel Feldmann and Claus Wagner

Institute of Aerodynamics and Flow Technology, DLR, Göttingen, Germany

e-mail: daniel.feldmannedlr.de 
more insight regarding the gas transport, efforts on experimental investigations have increased considerably in recent years, see e.g. the work by Adler \& Brücker [1] and Krenkel et al. [5]. The high complexity of pulmonary air flow due to very small and irregular geometries with poor or even without optical access makes numerical investigations essential to supplement such experiments. A lot of numerical studies were conducted but only a few considering high frequencies. The most recent and most comprehensive one was done by Choi et al. [2] using time varying Dirichlet boundary conditions (BC) for the velocity with a prescribed profile. In HFOV the flow field in the conducting airway region is governed by repetitive flow reversals and co-axial counter flows. Additionally, the irregular and multi-bifurcating geometry makes the flow and its impact on gas transport even more complex. Since the velocity distribution in the bronchi is a priori unknown, we use Dirichlet BCs for the pressure at all open boundaries and do not predefine a certain velocity profile, in contrast to the aforementioned studies. Thus, we assume Dirichlet pressure BCs to be more realistic. Furthermore, this allows for the setting of non-harmonic pressure waves, which is more similar to the flow driving mechanism of common HFOV devices.

\section{Theoretical Background}

To describe the oscillatory flow in the conducting airways we use the continuity equation and the unsteady Navier-Stokes equations in incompressible form

$$
\partial_{j} u_{j}=0 \quad \text { and } \quad \partial_{t} u_{i}+u_{j} \partial_{j} u_{i}=-\rho^{-1} \partial_{i} p+v \partial_{j}\left(\partial_{j} u_{i}+\partial_{i} u_{j}\right),
$$

where $\mathbf{u}$ is the velocity vector. Throughout this paper the working fluid is air which is inhaled at ambient temperature and then heated up to body temperature. Hence, we use a density of $\rho=1.15 \mathrm{~kg} / \mathrm{m}^{3}$ and a kinematic viscosity of $v=1.63 \times 10^{-5} \mathrm{~m}^{2} / \mathrm{s}$ corresponding to air at an average temperature of $30^{\circ} \mathrm{C}$ and a pressure of $p=1 \mathrm{bar}$. For an oscillatory fluid motion such as respiratory flow, the governing non-dimensional parameters are, according to e.g. Trukenmüller [8], the Womersley and the Reynolds number, defined as

$$
W o=D / 2 \cdot \sqrt{\omega v^{-1}} \quad \text { and } \quad R e=\hat{u} D v^{-1},
$$

respectively. Here, $D$ is a characteristic bronchi diameter and $\omega=2 \pi f$ is the ventilation frequency ranging from $f=0.2 \mathrm{~Hz}$ for quiet breathing up to $f=15 \mathrm{~Hz}$ for HFOV based respiration. As characteristic velocity we use the peak velocity $\hat{u}=\max \bar{u}(t)$ in $0<t \leqslant T$, where $\bar{u}$ is the instantaneous area-averaged mean velocity.

For a generic single bronchus, i.e. in our case a simple pipe geometry as sketched in fig. 1, we assume a laminar and axially symmetric flow which is also considered to be fully developed. By this we model a low Re flow far away from the inlets as well as periodic in $T$. Despite the fact that all bronchial tubes are too short and branch up too rapidly to maintain a fully developed flow at all, this approximation is 
Fig. 1 Schematic of a generic bronchus showing a laminar velocity profile (blue) of the oscillatory ventilation driven by a sinusoidally varying axial pressure gradient.

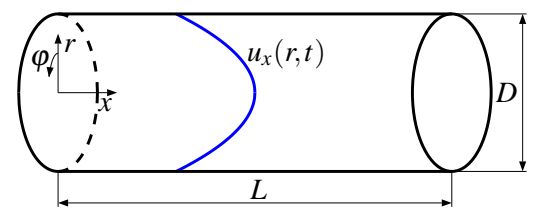

suitable as validation case for our flow simulations employing the Dirichlet pressure BCs. In both, spontaneous breathing and most HFOV, the flow is driven by a time varying axial pressure gradient

$$
-\rho^{-1} \partial_{x} p=f(t) \quad \Rightarrow \quad \frac{p_{x=0}(t)-p_{x=L}}{\rho L}=C \cdot \sin (\omega t) \quad \text { with } \quad C=\frac{-\hat{p}}{\rho L}
$$

which we presume to be oscillating in a sinusoidal manner. The restriction to harmonic wave forms is a common simplification for experimental and numerical investigations holding for spontaneous breathing, whereas most HFOV devices rather apply non-harmonic pressure waves. Using a complex ansatz for eq. (3) and the assumptions from above with eq. (1), one finally obtains

$$
u_{x}(r, t)=\Re\left\{\omega C^{-1}\left[1-J_{0}\left(r \sqrt{i \omega v^{-1}}\right) J_{0}\left(D / 2 \sqrt{i \omega v^{-1}}\right)^{-1}\right] e^{i \omega t}\right\}
$$

for the axial velocity. Eq. (4) originates to the work of Sexl [7] and Womersley [10] and is often used in the context of pulmonary and vascular fluid dynamics. Here, $i$ denotes the imaginary unit and $J_{0}$ the Bessel function of first kind and zeroth order. Since eq. (4) is complex, only the real part $\Re$ is of physical relevance.

Fig. 2 shows velocities for the full range of frequencies, which strongly affect the flow character. For $W o \ll 1$ the flow appears to be quasi steady, which means the fluid is able to instantly follow the driving force and therefore, the velocity develops a Hagen-Poiseuille profile at any time. With increasing Wo this shape gradually disappears, the profile deforms and stronger gradients develop close to the wall.

Fig. 2 Normalised profiles of the axial velocity (blue) in a generic bronchus for a wide range of non-dimensional frequencies $(W o)$ at eight different phases with respect to the oscillatory driving force (red) as obtained from the classical Sexl-Womersley (SW) solution of the incompressible Navier-Stokes equation for oscillatory laminar pipe flows, given in eq. (4). The amplitude of the driving pressure gradient is denoted by $C$.

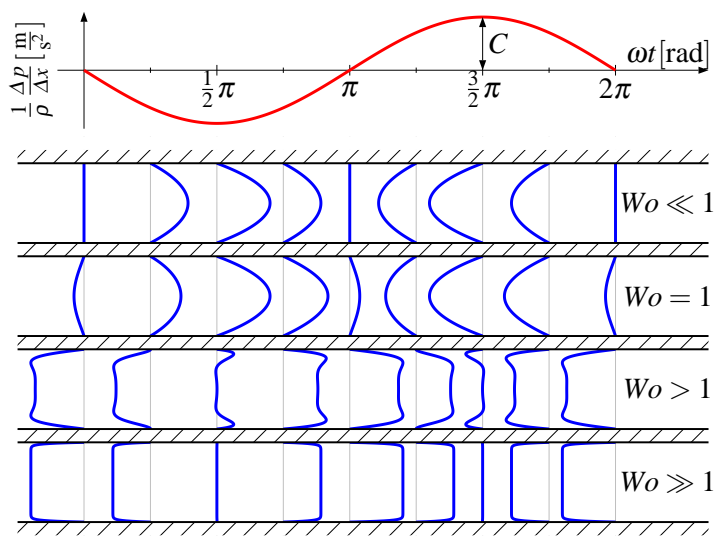


Furthermore, positive and negative axial velocity components occasionally co-exist, when flow reversal takes place from inspiration to expiration and vice versa. This is also referred to as co-axial counter flow and is related to a phase shift of the near wall flow with respect to the core flow. For $W o \rightarrow \infty$ the mean velocity $\bar{u}$ develops a phase lag with respect to the driving force, which approaches $\pi / 2$.

The respiratory system forms a complex geometry with airways branching out in $\sim 23$ generations comprising $>10^{7}$ single bronchi in total. According to Weibel [9], the characteristic length scales range from roughly $18 \mathrm{~mm}$ at the trachea down to $420 \mu \mathrm{m}$ at the alveolar level. Since in HFOV typical tidal volumes $V_{\text {tid }}$ and frequencies also cover a broad range of ventilator settings, it is even more important to know the non-dimensional parameter regime. The most commonly used HFOV devices are pressure-cycle machines that neither monitor nor directly control $V_{t i d}$ and the flow rate $\partial_{t} V$, respectively. Moreover, the influence of the conducting parts of the ventilator device and the endo-tracheal tube (ETT) are more or less unknown and so are the actual flow rates delivered to the lung opening during HFOV. Using a hot-wire anemometer Hager et al. [4] performed volume measurements in ARDS patients to characterise the effects of ventilator settings on $V_{\text {tid }}$. From this data we calculate the flow rate via $\left.\partial_{t} V\right|_{\max }=\pi \cdot V_{\text {tid }} / T_{\text {in }}$ under assumption of sinusoidal delivery to the lung in $T_{i n}=T / 3$ for $I: E=0.5$ and in $T_{i n}=T / 2$ for $I: E=1$, respectively. Herewith, we estimate the parameter range, shown in fig. 3 , for the whole bronchial tree and four clinically relevant respirator settings compared to a spontaneous breathing condition at peak inspiration. Each graph represents one scenario and each symbol represents $R e$ and $W o$ in a bronchial tube at one specific generation. The right most symbols denote the trachea, the left most denote the lowest terminal bronchioles and the single ones denote the corresponding situation in the ETT. The peak flow in each bronchus results from the simple assumption, that the flow rate subdivides according to the continuity eq. (1) and the total cross sectional area at each generation for a branching system of rigid pipes according to the classical airway model of Weibel [9]. To rate the parameter range in terms of transition to turbulence, fig. 3 additionally shows experimental results from Eckmann et al. [3] and findings from linear stability analysis by Trukenmüller [8] for pure oscillatory pipe flow. Furthermore, the parameter regime of the aforementioned numerical study from Choi et al. [2] is given.

Fig. 3 Complete range of the key dimensionless parameters in the entire bronchial tree under several respiratory conditions (graphs) and at every single generation (symbols) including ETT. The frequency, the tidal volume and the ratio $(I: E)$ of inspiration $\left(T_{i n}\right)$ to expiration $\left(T_{e x}\right)$ phase of each scenario are given in parentheses.

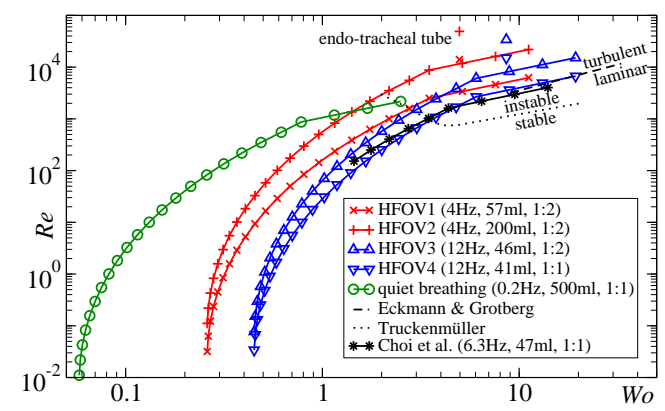


To solve the governing eq. (1) in discretised form, we use an unstructured finite volume method. For temporal integration an implicit second order backward differencing scheme is used, while the spatial discretisation is handled by an unbounded second order central differencing scheme. The solver takes advantage of the PISO method for pressure-velocity coupling and the resulting linear systems for the momentum equation and the Poisson equation are processed with a preconditioned biconjugate gradient solver and a geometric-algebraic multigrid solver, respectively. We already mentioned, that the flow in the conducting airways is rather complex and, therefore, the velocity profiles at the open boundaries are a priori unknown. Thus, we use pure Dirichlet BCs for the pressure and do not predefine a certain velocity profile at the open boundaries, which are in the case of HFOV all of these, inflow, outflow as well as mixed boundaries. In case of a single bronchus this leads to $\left.p\right|_{0}=C \cdot \sin (\omega t)$ and $\left.p\right|_{L}=0$, respectively. For the velocity we apply a mixed Dirichlet-Neumann BC at all open boundaries using a case-by-case analysis of the inward facing momentum flux $\Phi$ across each cell face. A von Neumann condition, i.e. $\left.\partial_{x} u\right|_{0, L}=0$, is used for the outflow faces $(\Phi<0)$, whereas a velocity value is obtained from the face normal component of the internal cell value using $\left.u\right|_{0, L}=\mathbf{u} \cdot \mathbf{n}$ for inflow faces $(\Phi \geqslant 0)$. As a simplification at $r=D / 2$ the standard no-slip and impermeability conditions are applied, despite the fact, that the bronchial walls are covered with cilia, mucus et cetera, which might have a relevant impact on the flow structure in terms of separation, transition and relaminarisation. For all simulations, the variable time step is adapted to follow a Courant number of at least $C o \leqslant 0.3$ throughout the whole oscillation cycle.

\section{Numerical Results}

Here, we rate the numerical results for the generic bronchi using the analytical SexlWomersley (SW) solution. According to fig. 3, Wo $=14, W o=6$ and $W o=3$ fall in the typical range of HFOV in the first seven generations. Moreover, the latter also represents the situation in quiet breathing at the trachea. Radial velocity profiles are plotted in fig. 4 for $\omega t=\pi$ and the computed laminar flow fields are in excellent agreement with eq. (4). Note, that at this phase $\bar{u} \neq 0$, due to Wo well over unity and the resulting phase shift. Since the initial condition is $\bar{u}=0$, the rising pressure accelerates the air and causes an overshoot in $\hat{u}$. From fig. 4(a) to 4(c) we conclude, that for increasing $W o$, a significantly longer settling phase $t / T$ is required to let the flow converge to the SW solution, due to a shorter deceleration phase. However, utilising the deviation of the numerical from the analytical results, defined as

$$
\varepsilon(t)=\sum_{i=1}^{N_{r}}\left|u_{\text {mum }}^{*}\left(r_{i}, t\right)-u_{\text {man }}^{*}\left(r_{i}, t\right)\right| \Delta r_{i} \quad \text { with } \quad u^{*}(r, t)=\frac{u(r, t)}{\hat{u}_{\text {ama }}},
$$

we finally obtain an accuracy of at least $\varepsilon=10^{-2}$ for all Wo on a structured hexahedral mesh with $N_{r}=40$ equidistant grid points per radius. Nevertheless, HFOV 


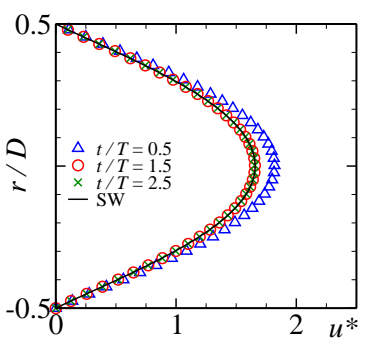

(a) $W o=3, R e=620$

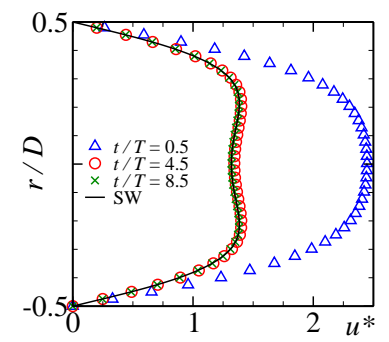

(b) $W o=6, R e=160$

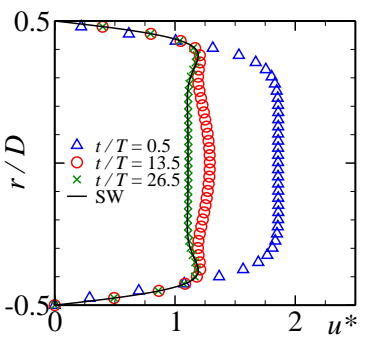

(c) $W o=14, R e=40$

Fig. 4 Velocities obtained by numerical simulations in a generic bronchus for several Wo from breathing to HFOV after three different numbers of cycles compared to the SW solution (eq. (4)).

simulations involving high Wo take about ten times more oscillation cycles to reach equilibrium when compared to spontaneous breathing simulations. We further found that applying an initial bulk velocity $\bar{u}$ corresponding to eq. (4) at $t=0$, reduces the settling phase by a factor of two. Initialising the exact SW profile would, of course, completely avoid this problem in the case of a straight pipe, but does not work for irregular and multi-bifurcating airway geometries. Even though, the pressure amplitude (3) is identical for all three simulations, higher Wo lead to a several times lower $\hat{u}$. This is quantified by the decreasing Re shown in fig. 4(a) to 4(c) and is also known from clinical practise, see e.g. Hager et al. [4]. The rightmost term of eq. (4) is more or less a periodic function of Wo being responsible for the profile's shape, whereas the leftmost factor scales the profile according to the pressure amplitude $C$ and the frequency $\omega$. As stated earlier, this leads to much more deformed velocity profiles for increasing Wo. Therefore, HFVO simulations require finer grids to adequately resolve the stronger gradients $\partial_{r} u_{x}$ occurring in the vicinity of the wall despite of relatively low $R e$.

We also apply the Dirichlet pressure BC to a symmetric bifurcation, employing the oscillating value at the single parent tube. The computational domain consists of a $55^{\circ}$ bifurcation and three tubes of ten diameters in length discretised with $5.5 \times 10^{5}$ tetrahedral and hexahedral grid cells. To demonstrate stable runs of oscillatory simulations comprising more than one additional open $\mathrm{BC}$, we use identical as well as slightly different but constant pressure values at the distal ends of the two daughter tubes. The latter case is also a first approach to take uneven physiological behaviour of two different lung regions adjacent to the lower bifurcation ends into account. Fig. 5 shows flow fields obtained from numerical simulations for $W o=14$, $R e=620$ and three different pressure BCs with the exact values given in the caption. Since the velocity is plotted for $\omega t=3 \pi / 20$, i.e. for early inspiration, we can observe the co-axial counter flow phenomena in fig. 5(a), as expected from the SW solution for a generic bronchus. Here, the inspiratory flow near the wall is indicated by the blue and the expiratory flow in the core regions is indicated by red colour, using

$$
|\mathbf{u}| \cdot \frac{\mathbf{u} \cdot \mathbf{n}}{|\mathbf{u} \cdot \mathbf{n}|},
$$




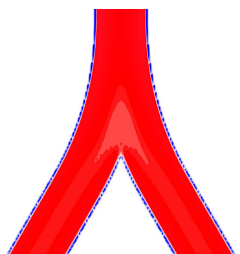

(a) $p_{l}=p_{r}=0$

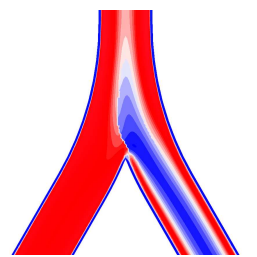

(b) $p_{l}=0, p_{r}=\frac{-C}{1000}$ (c) $p_{l}=0, p_{r}=\frac{-C}{100}$
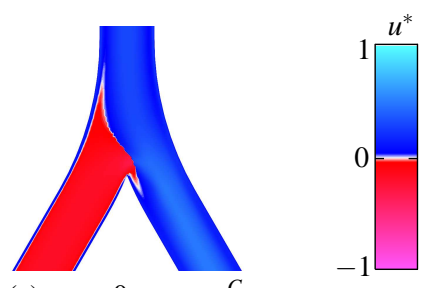

Fig. 5 Colour encoded velocity plots in the coronal plane of the bifurcation at $\omega t=3 \pi / 20$ for $W o=14$ and three different pressure BCs at the lower left $\left(p_{l}\right)$ and right $\left(p_{r}\right)$ end, respectively.

where $\mathbf{n}$ is the vector normal to the local cross sectional area. The flow field is completely symmetric for identical outlet pressure values and becomes more asymmetric with increasing pressure differences, as reflected in fig. 5(b) and 5(c). In this case some fluid flows from the left daughter branch into the right one, getting more distinct for increasing $\Delta p$. Similar flow structures can also occur in healthy and, more so, in diseased lungs due to uneven physiological behaviour of different airway regions in terms of variation in regional airway resistance or compliance.

Finally, we present results of the flow in a three-generation lung geometry with multiple straight outlets reconstructed from computer tomography (CT) image data. The oscillating $\mathrm{BC}$ is applied to the trachea while the additional nine bronchial ends maintain identical fixed pressure values. The computational domain consists of approximately $1.4 \times 10^{6}$ tetrahedral grid cells. Again, we use a typical HFOV frequency, resulting in $W o=14$ and $R e=120$ at the trachea, whereas actual $R e$ in HFOV are considerably higher, as can be seen from fig. 3. Fig. 6 shows the airway geometry, the pressure distribution and the flow field in the trachea for $\omega t=3 \pi / 2$, when the driving pressure reaches its minimum and early expiration takes place. Here, a phase shift does also occur between the driving pressure and $\bar{u}$ due to Wo well over unity. Thus, we observe expiratory flow (red) close to the airway walls and an inspiratory core flow (blue) at the same time, using eq. (6). The co-axial counter flow continuously exists throughout the whole geometry from the trachea down to lowest bronchi.

Fig. 6 Colour encoded pressure and velocity distributions in the CT lung model (left) and a sagittal cross section of the trachea (right) for $W o=14$ and $R e=120$ at $\omega t=3 \pi / 2$, when flow reversal from inspiration (blue) to expiration (red) takes place, see eq. (6).
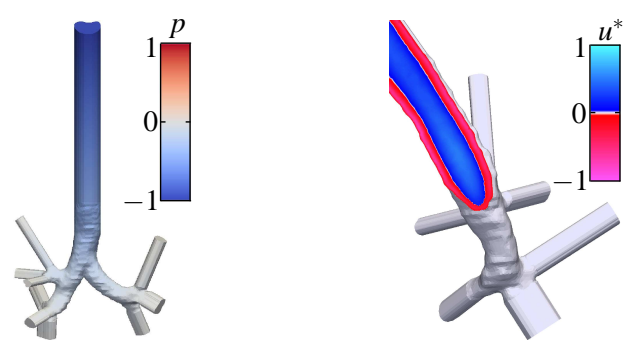


\section{Concluding Remarks}

HFOV is an established artificial respiration technique in neonatalogy, providing potential benefits for the treatment of ARDS in adult patients. However, the advantages have not yet been translated into improved outcomes when compared to conventional ventilation, due to a lack of insight into the gas transport mechanisms. To improve this we performed numerical simulations of the oscillatory flow in simplified airway geometries using time varying Dirichlet BCs for the pressure and a second order accurate finite volume method. In the present contribution we presented numerical results of the oscillatory flow similar to that under conditions of HFOV in a straight pipe, a symmetric bifurcation and a three-generation airway geometry reconstructed from CT image data. It was demonstrated, that the use of Dirichlet pressure BCs allows to adequately reproduce the expected flow fields. For future simulations this allows the use of more realistic pressure BCs, such as nonharmonic pressure waves at the upper boundary, representing the HFOV device, and flow rate dependent pressure $\mathrm{BCs}$ at the distal ends at the same time.

Acknowledgements The authors acknowledge financial support by the German Research Foundation under grant WA 1510/8. DF would also like to thank S. Horn for many useful conversations.

\section{References}

1. Adler, K., Brücker, C.: Dynamic flow in a realistic model of the upper human lung airways. Exp. Fluids 2-3, 411-423 (2007)

2. Choi, J., Xia, G., Tawhai, M., Hoffman, E., Lin, C.L.: Numerical study of high-frequency oscillatory air flow and convective mixing in a CT-based human airway model. Ann. Biomed. Eng. 38, 3550-3571 (2010)

3. Eckmann, D.M., Grotberg, J.B.: Experiments on transition to turbulence in oscillatory pipe flow. J. Fluid Mech. 222, 329-350 (1991)

4. Hager, D., Fessler, H., Kaczka, D., Shanholtz, C., Fuld, M., Simon, B., Brower, R.: Tidal volume delivery during HFOV in adults with ARDS. Crit. Care Med. 35, 1522-1529 (2007)

5. Krenkel, L., Pennecot, J., Lenz, C., Feldmann, D., Wagner, C.: Rekonstruktion von Geometrien aus medizinischen Bilddaten und Erstellung von Modellen für experimentelle Strömungsuntersuchungen. In: Dresdner Beiträge zur Medizintechnik, vol. 10. TUDpress (2010)

6. Krishnan, J.A., Brower, R.G.: High-frequency ventilation for acute lung injury and ARDS. CHEST 118(3), 795-807 (2000)

7. Sexl, T.: Über den von E. G. Richardson entdeckten Annulareffekt. Z. Phys. A 61, 349-362 (1930)

8. Trukenmüller, K.E.: Stabilitätstheorie für die oszillierende Rohrströmung. Ph.D. thesis, Helmut-Schmidt-Universität, Hamburg (2006)

9. Weibel, E.R.: Morphometry of the human lung. Academic Press, New York (1963)

10. Womersley, J.R.: Method for the calculation of velocity, rate of flow and viscous drag in arteries when the pressure gradient is known. J. Physiol. 127, 553-563 (1955) 